\title{
An effective electron-electron interaction in semi-infinite metal in the presence of external static electric field with taking into account the local field approximation
}

\author{
Markovych B., Zadvorniak I. \\ Lviv Polytechnic National University \\ 12 S. Bandera str., 79013, Lviv, Ukraine
}

(Received 1 September 2016)

\begin{abstract}
The electron-electron interaction in semi-infinite metal is considered. The influence of local field approximation and the influence of external static electric field strength on two-particle correlation function and effective potential of electron-electron interaction are investigated.
\end{abstract}

Keywords: two-particle correlation function, effective potential of electron-electron interaction, external static electric field strength, jellium model.

2000 MSC: $82 \mathrm{~B} 24$

UDC: 530.145

\section{Introduction}

Many-particle correlation functions effective potential of electron-electron interaction $[1,2]$ are necessary for constructing quantum-statistical theory of semi-bounded metal. In particular, these functions are needed to calculate thermodynamic and structural characteristics of metal. Many authors [3-7] investigated electron-electron interaction in metals, especially near the metal surface.

The task of the investigation of the influence of external static electric field on surface effects of metals has actually been set in works [8-10]. Thus in [8], it was investigated for the influence of electric field on anisotropy of surface energy of alkali metal alloy using the density function theory (DFT). DFT [11] is an effective mathematical approach for description of the electron structure of many-particle systems. However, it is a one-particle approach, so it cannot consider many-particle correlations effects correctly.

An effective electron-electron interaction in semi-infinite metal in the presence of static electric field applied perpendicular to the surface is investigated in present work. The spatial heterogeneity of unperturbed electrons density and the local field approximations are considered during the investigation. A calculation of two-particle correlation function and pair effective potential of electron-electron interaction for semi-bounded metal in the jellium model according to the approach proposed in $[12,13]$ is realized in present work. It allows us to use different forms of local field approximations proposed by Hubbard [14], Ichimaru [15] and others, which were used for homogeneous electron gas.

\section{Model}

We consider the system of $N$ electrons in the space

$$
\Omega=\{(x, y, z):-\sqrt{S} / 2 \leqslant x \leqslant \sqrt{S} / 2,-\sqrt{S} / 2 \leqslant y \leqslant \sqrt{S} / 2,-L / 2 \leqslant z \leqslant L / 2\}
$$

in the field of a positive inhomogeneously distributed charge $\rho(\boldsymbol{R})=\rho\left(\boldsymbol{R}_{\|}, Z\right)$, where $\boldsymbol{R}_{\|}=(X, Y)$. A semi-bounded metal is described within framework of the jellium model. The division surface "metalvacuum" is plane and described by the equation $Z=-d=$ const . 
For this model $\rho_{\text {jell }}(\boldsymbol{R})=\rho_{\text {jell }}(Z)=\rho_{0} \theta(-d-Z), \rho_{0}=\frac{e N}{S L / 2}$ and the surface potential is a function of only normal electron coordinate to the division surface $V(\boldsymbol{r}) \equiv V(z)$.

The surface potential is modelled by the expression

$$
V(z)= \begin{cases}W+e E z, & z>0 \\ 0, & z<0\end{cases}
$$

where $E$ is the strength of external electric field applied perpendicular to the division surface "metalvacuum" in the metal direction, $W=\hbar^{2} s^{2} /(2 m), W$ is the barrier height, $e$ is the electron charge. For $E=0$, the surface potential (1) takes the form of the fixed height step. For $E \rightarrow \infty$, the potential $V(z) \rightarrow \infty$. In this case, the external electric field does not let the electrons leave the metal. So, it became the analog of the infinity height potential barrier model.

An effective potential of electron-electron interaction

$$
g\left(\boldsymbol{r}_{\|}, z_{1}, z_{2}\right)=\sum_{\boldsymbol{q}} e^{i \boldsymbol{q} \boldsymbol{r}_{\|}} g\left(q \mid z_{1}, z_{2}\right)
$$

The Fourier image $g(q \mid z, z)$ of the effective potential is determined by the following expression [12]:

$$
g\left(q \mid z_{1}, z_{2}\right)=V\left(q \mid z_{1}-z_{2}\right)+\frac{\beta}{S L^{2}} \int d z \int d z^{\prime} V\left(q \mid z_{1}-z\right) \tilde{\mathfrak{M}}\left(q \mid z, z^{\prime}\right) V\left(q \mid z^{\prime}-z_{2}\right),
$$

where $V\left(q \mid z_{1}-z_{2}\right)=\frac{2 \pi e^{2}}{q} e^{-q\left|z_{1}-z_{2}\right|}$ is a two-dimensional Fourier image of Coulomb potential, $z_{1}, z_{2}$ are normal coordinates of electrons to the division surface, $\beta$ is inverted thermodynamic temperature, $\tilde{\mathfrak{M}}\left(q \mid z, z^{\prime}\right)$ is two-particle correlation function "density-density" that is the solution of second order Fredholm integral equation [13]:

$$
\tilde{\mathfrak{M}}\left(q \mid z_{1}, z_{2}\right)=\mathfrak{M}_{0}\left(q \mid z_{1}, z_{2}\right)+\frac{\beta}{S L^{2}} \int d z \int d z^{\prime} \mathfrak{M}_{0}\left(q \mid z_{1}, z\right)\left\{v\left(q \mid z-z^{\prime}\right)-\bar{v}\left(q \mid z-z^{\prime}\right)\right\} \tilde{\mathfrak{M}}\left(q \mid z^{\prime}, z_{2}\right),
$$

where $\bar{v}_{k}(q)=G_{k}(q) v_{k}(q), \bar{v}\left(q \mid z-z^{\prime}\right)=\frac{1}{L} \sum_{k} e^{i k\left(z-z^{\prime}\right)} \bar{v}_{k}(q), \mathfrak{M}_{0}\left(q \mid z_{1}, z\right)$ is two-particle correlation function "density-density" in random phases approximation (RPA) [13], $G_{k}(q)$ is matrix of local field approximation element. The calculation of two-particle correlation function (3) and pair effective potential of electron-electron interaction (2) is conducted for different forms of local field approximation.

\section{Numerical results}

The research was performed for different strength of electric field (in particular for the cases $E=0$ and $E \rightarrow \infty$ ) for the following local field approximations:

1) Hubbard approximation [14] $G_{k}(q)=\frac{1}{2} \frac{q^{2}+k^{2}}{q^{2}+k^{2}+p_{\mathrm{F}}^{2}}$, and also modified Hubbard approximation:

$$
G_{k}(q)=\frac{1}{2} \frac{q^{2}+k^{2}}{q^{2}+k^{2}+\xi p_{\mathrm{F}}^{2}}
$$

where $p_{\mathrm{F}}=\frac{\sqrt{2 m \mu}}{\hbar}, \mu$ is the chemical potential of electron subsystem, $m$ is the electron mass, $\xi$ is the parameter, the values of which are: at $\xi=0$, the approximation (4) is Hartri approximation, at $\xi=1$ is Hubbard approximation, at $\xi=2$ is Geldart and Vosko approximation, at $\xi=1+\frac{2}{\pi p_{\mathrm{F}} a_{\mathrm{B}}}$ is Animalu approximation, at $\xi=1+\frac{4}{\pi p_{\mathrm{F}} a_{\mathrm{B}}}$ is Sham approximation for homogeneous electron gas.

2) Ichimaru approximation [15]

$$
G_{k}(q)=A Q^{4}+B Q^{2}+C+\frac{4-Q^{2}}{4 Q} \cdot\left[A Q^{4}+\left(B+\frac{8}{3} A\right) Q^{2}-C\right] \cdot \ln \left|\frac{2+Q}{2-Q}\right| .
$$

Mathematical Modeling and Computing, Vol. 3, No. 1, pp.90-96 (2016) 
Here $Q=\frac{\sqrt{q^{2}+k^{2}}}{p_{\mathbb{F}}} ; A=0.029, B=\frac{9}{16} \gamma_{0}-\frac{3}{16}[1-g(0)]-\frac{16}{15} A, C=-\frac{3}{4} \gamma_{0}+\frac{9}{16}[1-g(0)]-\frac{16}{5} A$; $g(0)=\frac{1}{8}\left[\frac{z}{I_{1}(z)}\right]^{2}, I_{1}(z)$ is modified Bessel function of the first kind;

$$
\gamma_{0}=\frac{1}{4}-\frac{\pi \alpha}{24} r_{s}^{5} \frac{d}{d r_{s}}\left(\frac{b_{0}}{r_{s}^{3}} \cdot \frac{1+b_{1} \sqrt{r_{s}}}{1+b_{1} \sqrt{r_{s}}+b_{2} r_{s}+b_{3} r_{s}^{3 / 2}}\right)
$$

$\alpha=\left(\frac{4}{9 \pi}\right)^{1 / 3}, p_{F}=(9 \pi / 4)^{1 / 3} / r_{s}, r_{s}$ is Bruckner's parameter; $b_{0}=0.06218, b_{1}=9.81379, b_{2}=2.82224$, $b_{3}=0.73641$.

The research is performed for potassium (the Brueckner parameter $r_{s}=4.86$ [16], the lattice period $5.225 \AA$ or $\left.9.87 a_{\mathrm{B}}[17]\right)$.

\section{Numerical results of two-particle correlator $\tilde{\mathfrak{M}}\left(q \mid z_{1}, z_{2}\right)$}

Two-particle correlation function $\tilde{\mathfrak{M}}\left(q \mid z_{1}, z_{2}\right)$ is a solution of the equation (3). In Figs. 1-3, the dependencies of the two-particle "density-density" correlation function $\tilde{\mathfrak{M}}\left(q \mid z_{1}, z_{2}\right)$ on coordinate of one electron $\left(z_{2}\right)$ normal to the surface is depicted. The corresponding coordinate of the other electron $\left(z_{1}\right)$, the values $q$ and $E$ are fixed. The investigation was carried out for different values of $E$ and $q$ and also for different forms of local field approximation.

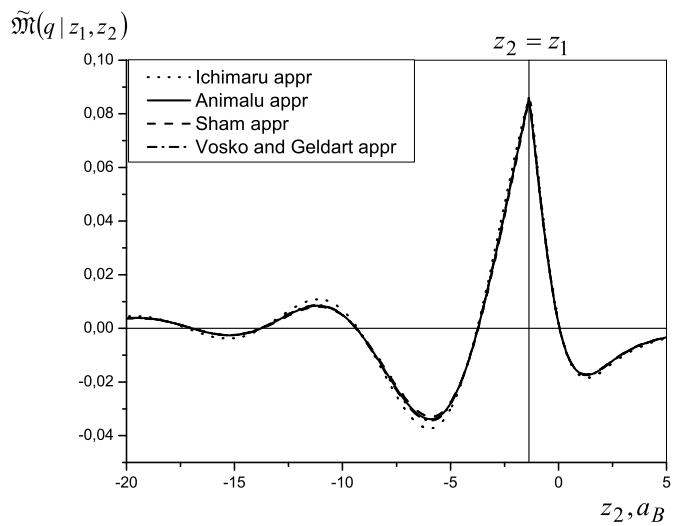

Fig. 1. $E=10 \mathrm{~V} / \mathrm{nm}, z_{1}=-1.37 a_{\mathrm{B}}, q=0.0185 a_{\mathrm{B}}^{-1}$.

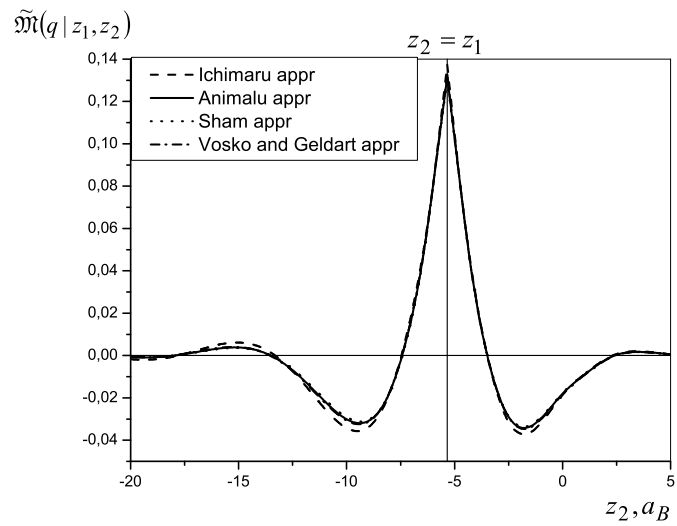

Fig. 3. $E=10 \mathrm{~V} / \mathrm{nm}, z_{1}=-5.34 a_{\mathrm{B}}, q=0.0185 a_{\mathrm{B}}^{-1}$.

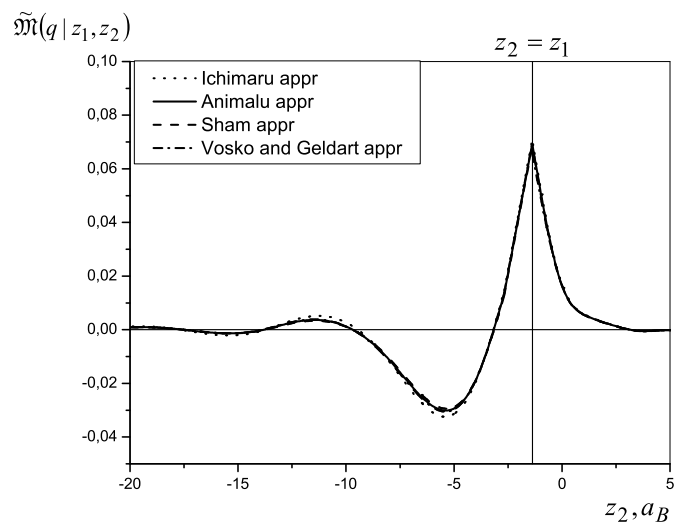

Fig. 2. $E=20 \mathrm{~V} / \mathrm{nm}, z_{1}=-1.37 a_{\mathrm{B}}, q=0.0185 a_{\mathrm{B}}^{-1}$.

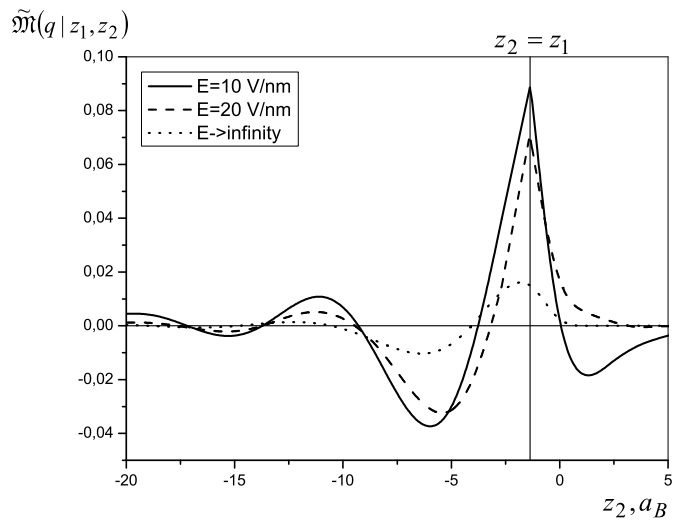

Fig. 4. $\tilde{\mathfrak{M}}\left(q \mid z_{1}, z_{2}\right)$ for Ichimaru approximation for the fixed coordinates $z_{1}$ and $q$ and different values of strength. $z_{1}=-1.37 a_{\mathrm{B}}, q=0.0185 a_{\mathrm{B}}^{-1}$. 
Figs. 1-3 demonstrate that extremums of the function $\tilde{\mathfrak{M}}\left(q \mid z_{1}, z_{2}\right)$ are slightly shifted. It is observed for different values of strength and also for different coordinates.

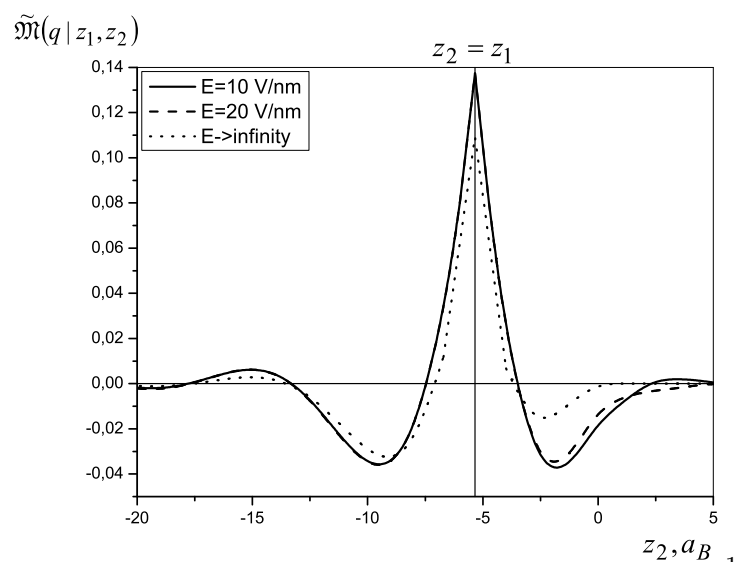

Fig. 5. Ditto for $z_{1}=-5.34 a_{\mathrm{B}}, q=0.0185 a_{\mathrm{B}}^{-1}$.

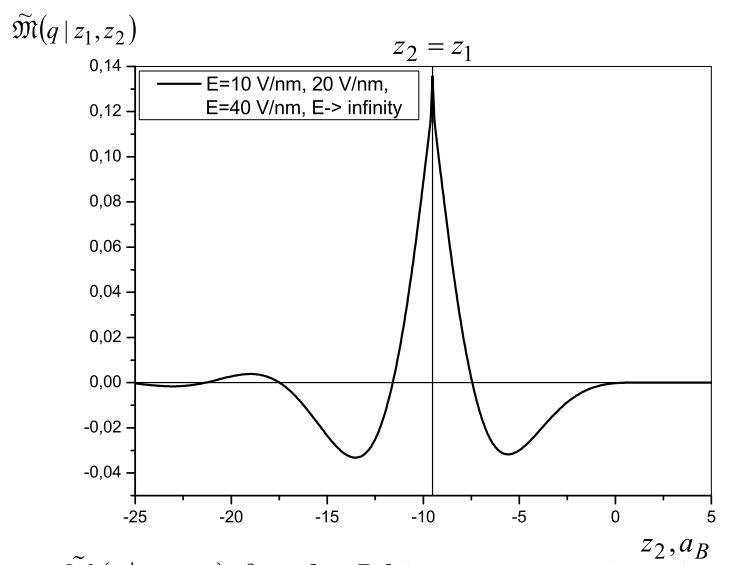

Fig. 6. $\tilde{\mathfrak{M}}\left(q \mid z_{1}, z_{2}\right)$ for the Ichimaru approximation at $z_{1}=-9.52 a_{\mathrm{B}}, q=0.0185 a_{\mathrm{B}}^{-1}$ and different values of strength.

Figs. 4,5 show us the dependencies of the two-particle "density-density" correlation function $\tilde{\mathfrak{M}}\left(q \mid z_{1}, z_{2}\right)$ on the coordinate of one electron $\left(z_{2}\right)$ normal to the surface, whereas the corresponding coordinate of the second electron $\left(z_{1}\right)$ and the value $q$ are fixed. These dependencies are obtained for Ichimaru approximation and different values of $E$. Fig. 4 demonstrates that the increase in the intensity leads to the decrease of the maximum of the function $\tilde{\mathfrak{M}}\left(q \mid z_{1}, z_{2}\right)$ and the extremums of function $\tilde{\mathfrak{M}}\left(q \mid z_{1}, z_{2}\right)$ are slightly different for different values of $E$. The influence of external electric field on electrons correlation is significant near the surface (in distances from the surface $1-2 a_{\mathrm{B}}$ ). Obtained dependencies for the distances from the surface equal approximately a half of period of lattice $\left(5 a_{\mathrm{B}}\right)$ are only little shifted (see Fig. 5). So, the influence of external electric field on $\tilde{\mathfrak{M}}\left(q \mid z_{1}, z_{2}\right)$ is minor. The dependencies for different $E$ are matched in Fig. 3. It occurred at $z_{1}=-9.52 a_{\mathrm{B}}$, i.e. for the distances from the surface equal approximately one period of lattice of potassium $\left(9.87 a_{\mathrm{B}}\right)$.

If one of electrons is in the metal bulk, the function $\tilde{\mathfrak{M}}\left(q \mid z_{1}, z_{2}\right)$ is symmetric (see Fig. 6) with respect to the coordinate of the fixed electron and has a sharp peak, when coordinates of two electrons coincide $\left(z_{2}=z_{1}\right)$, i.e. the electrons correlate with each other and are not influenced by the surface.

\section{Numerical results of effective potential of electron-electron interaction $g\left(r_{\|}, z_{1}, z_{2}\right)$}

After inverse Fourier transform of the effective potential (2), we obtain the expression for calculation of effective potential:

$$
g\left(r_{\|}, z_{1}, z_{2}\right)=\frac{1}{2 \pi} \int_{0}^{\infty} q J_{0}\left(q r_{\|}\right) g\left(q \mid z_{1}, z_{2}\right) d q,
$$

where $J_{0}(x)$ is Bessel function of zero kind.

The dependence of $g\left(r_{\|}, z_{1}, z_{2}\right)$ on the distance between the electrons along the surface is depicted in Figs. 7-9. The electron coordinates $z_{1}$ and $z_{2}$ normal to the surface are identical and fixed near the surface (for the distances from the surface that not exceed a half of period of lattice). The investigation was carried out for different values of $E$ and also for different forms of local field approximation. 


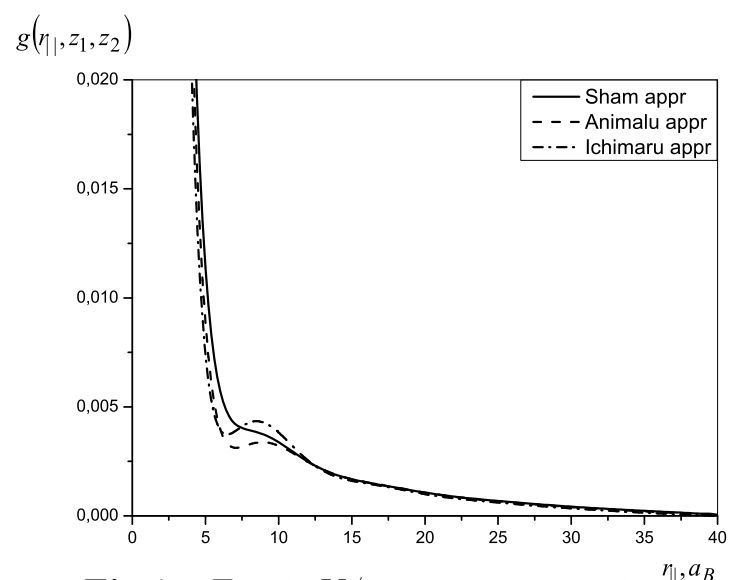

Fig. 7. $E=10 \mathrm{~V} / \mathrm{nm}, z_{1}=z_{2}=-1 a_{\mathrm{B}}^{\eta_{1}, a_{B}}$

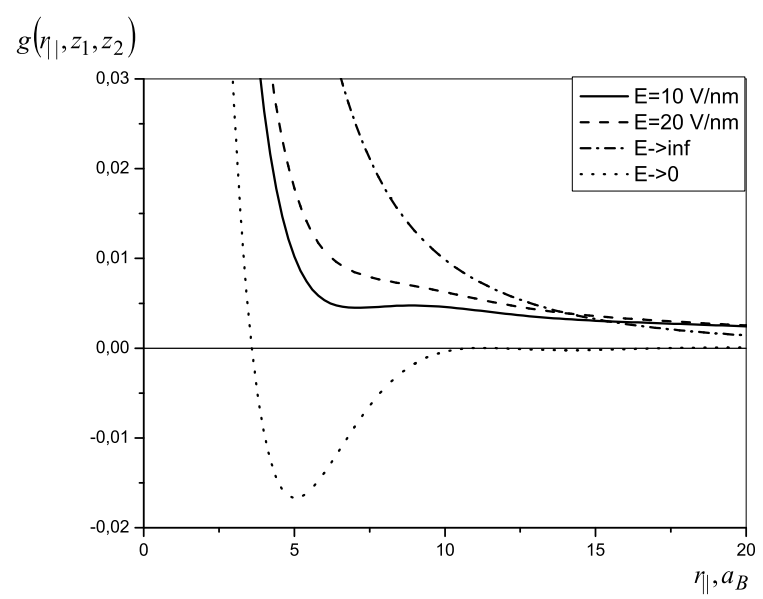

Fig. 9. $g\left(r_{\|}, z_{1}, z_{2}\right)$ for the Animalu approximation at $z_{1}=z_{2}=-1 a_{\mathrm{B}}$ and different values of strength.

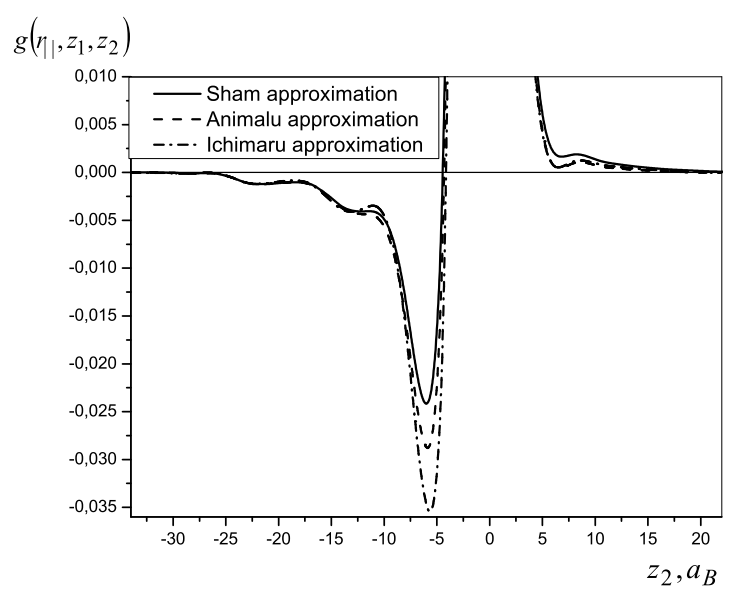

Fig. 10. $E=10 \mathrm{~V} / \mathrm{nm}, z_{1}=-1 a_{\mathrm{B}}, r_{\|}=0$.

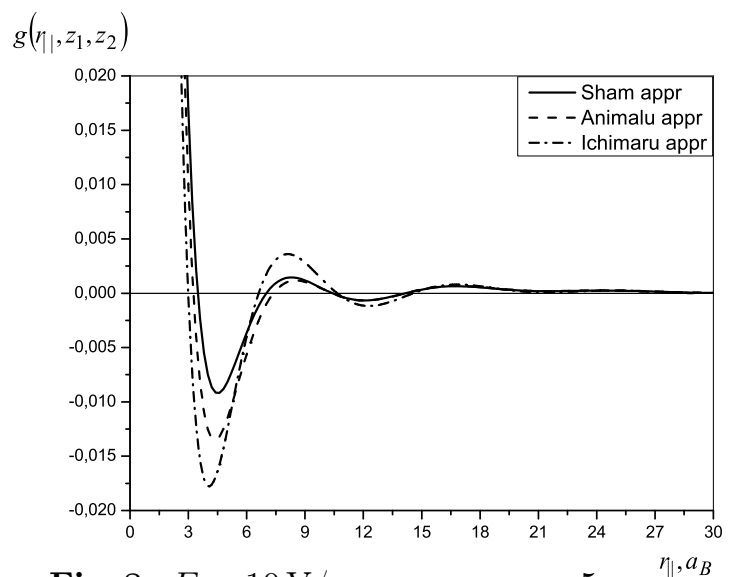

Fig. 8. $E=10 \mathrm{~V} / \mathrm{nm}, z_{1}=z_{2}=-5 a_{\mathrm{B}} \cdot{ }^{{ }_{\|}, a_{\mathrm{B}}}$

Figs. 7, 8 demonstrate that obtained dependencies are rather different for different approximations, particular the value of potential hole (see Fig. 8). In Fig. 9, the dependence of $g\left(r_{\|}, z_{1}, z_{2}\right)$ on $r_{\|}$for Animalu approximation and different values of $E$ is depicted. The calculations are also carried out for cases of $E \rightarrow \infty$ (infinity potential barrier) and $E \rightarrow 0$ (without external static electric field). Fig. 9 shows us that the increase in the intensity leads to the decrease of the value of potential hole.

In Figs. 10, 11, the dependence of $g\left(r_{\|}, z_{1}, z_{2}\right)$ on normal coordinate of one of the electrons $z_{2}$ is shown. The other electron coordinate $z_{1}$, the distance between the electrons along the surface $\left(r_{\|}\right)$and $E$ are fixed for the different local field approximations.

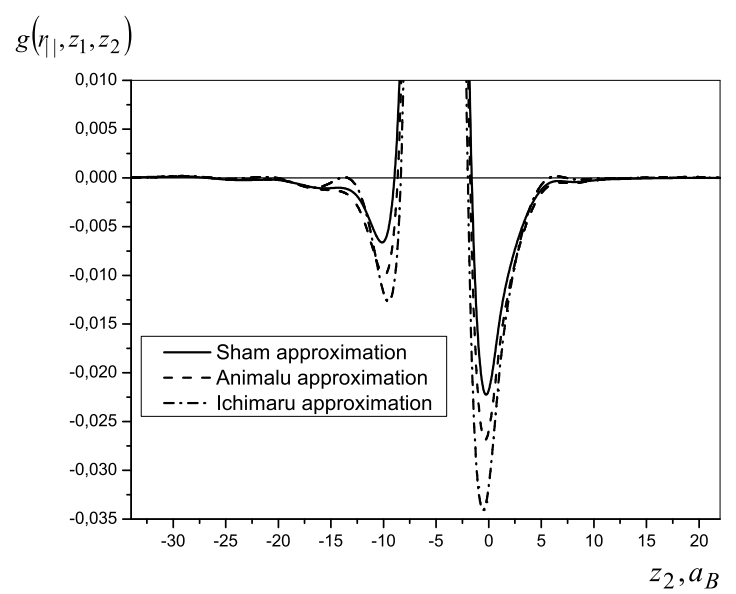

Fig. 11. $E=10 \mathrm{~V} / \mathrm{nm}, z_{1}=-5 a_{\mathrm{B}}, r_{\|}=0$.

Figs. 10,11 as well as Figs. 7,8 demonstrate that obtained dependencies are different for different approximations. 


\section{Conclusions}

The influence of the local field approximation and external static electric field on density distribution function and on pair effective potential of electron-electron interaction are investigated. It is shown that the choice of form of local field approximation leads to different results of these functions. That is why it is needed to choose accurately the local field approximation for the correct description of the system.

The numerical results shows us that near the surface (in the distances from the surface equal approximately one period of lattice) the value of effective potential is different for the different values of the external static electric field strength. In the metal depth (for the distances from the surface equal more than one period of lattice) the influence of the electric field strength does not appear. That is why the external electric field does not permeate on such distances from the surface. These results in the metal depth are also identical to the results obtained in [12] (effective potential) and [13] (two-particle correlator).

[1] Vavrukh M., Paslavskii V. Reference System Approach in the Electron Liquid Theory. Phys. Stat. Sol. B. 91, 208 (1998).

[2] Kostrobij P. P., Markovych B. M. Semi-infinite metal: perturbative treatment based on semi-infinite jellium. Condens. Matter Phys. 11, 641 (2008).

[3] Chakrav S., Fogel M., Kohn W. Dynamic response of inhomogeneous Fermi systems. Phys. Rev. Lett. 43, 775 (1979).

[4] Sahni V. Exchange charge density at metallic surfaces. Phys. Rev. B. 29, 1045 (1984).

[5] Eguiluz A. G., Kampbell D. A., Maradudin A. A, Wallis R.F. Static response of a jellium surface: The image potential and indirect interaction between two charges. Phys. Rev. Lett. 30, 5449 (1984).

[6] Kohn W., Mattsson A. E. Edge electron gas. Phys. Rev. Lett. 115, 3487 (1998).

[7] Ángyán J. G., Gerber I. C., Savin A., Toulouse J. Van der Waals forces in density functional theory: Perturbational long-range electron-interaction corrections. Phys. Rev. A. 72, 012510 (2005).

[8] Kanchukoev V.Z., Kashezhev A. Z., Mambetov A. H., Sozaev V. A. The influence of an electric field on the anisotropy of the surface energy of alkali metals alloys. JTF Lett. 27, 89 (2001).

[9] Zubarev N. M. Exact solutions for stationary profile of the liquid metal surface in the external Electric Field. JTF Lett. 25, 79 (1999).

[10] Guo W., Du S. X., Zhang Y. Y., Hofer W. A., Seidel C., Chi L. F., Fuchs H., Gao H.-J. Dynamic response of inhomogeneous Fermy systems. Surf. Sci. 603, 2815 (2009).

[11] Lang N. D., Kohn W. Theory of metal surfaces: charge density and surface energy. Phys. Rev. B. 1, 4555 (19790).

[12] Kostrobij P. P., Markovych B. M. An effective potential of electron-electron interaction in semi-infinite jellium. Condens. Matter Phys. 9, 747 (2006).

[13] Kostrobij P. P., Markovych B. M., Kazanovska O. Z. Preprint of the Institute for Condensed Matter Physics, ICMP-06-15U (2006).

[14] Gorobchenko V. D., Maksimov E. G. Dielectric permittivity of interacting electron gas. Physics-Uspekhi. 130, 65 (1980).

[15] Ichimaru S., Utsumi K. Analytic expression for the dielectric screening function of strongly coupled electron liquids at metallic and lower densities. Phys. Rev. B. 24, 7385 (1981).

[16] Vavruh M., Koval S., Tyshko N. Modelling of the short-range correlations in the degenerated electron systems. Visnyk LVIV UNIV. Ser Physic. 33, 7 (2000).

[17] Kittel C. Quantum theory of solids. John Wiley \& Sons, Inc., New York, London (1963). 


\title{
Ефективна взаємодія між електронами в напівобмеженому металі при наявності зовнішнього електричного поля з урахуванням поправки на локальне поле
}

\author{
Маркович Б. М., Задворняк I. М. \\ Національний університет «Львівська політехніка» \\ вул. С. Бандери, 12, 79013, Львів, Україна
}

Розглянуто міжелектронну взаємодію в напівобмеженому металі біля поверхні поділу. Досліджено вплив поправки на локальне поле та напруженості прикладеного зовнішнього електричного поля на двочастинкову кореляційну функцію електронів та ефективний потенціал міжелектронної взаємодії.

Ключові слова: двочастинкова кореляційна функція, ефективний потенціал міжелектронної взаємодіӥ, напруженість зовнішнъого статичного електричного поля, модель желе.

2000 MSC: $82 \mathrm{~B} 24$

УдК: 530.145

Mathematical Modeling and Computing, Vol.3, No.1, pp. 90-96 (2016) 